\title{
A brief review of application of Graphene in the field of Electrical sensor as field effect transistor
}

\author{
Reshmi Bose ${ }^{\mathrm{a}^{*}}$ \\ ${ }^{a}$ Department of BSH, University of Engineering \& Management (UEM), Kolkata \\ * Corresponding Author : reshmi.bose@uem.edu.in
}

\section{Abstract:}

Graphene is considered to be the most efficient material for the future of nanomaterials. Due to its unique structure and good thermal and electrical conductivity opened a door for future research. The graphene based field effect transistors are one of the most important application of graphene. Thus it can be used after $\mathrm{p}$ or $\mathrm{n}$ doping for the detection and analysing some of the substrate. Thus this paper contains a very brief review on the graphene in field of electrical sensor.

\section{Introduction:}

The advancement of Nanotechnology opens a new door for material based nano science providing a lot of exciting opportunities in the field of biotechnological development due to their special structure, components and properties [1]. This particular branch of science establish the real evidence for the statement of Leonardo da Vinci who said 'Where nature finishes producing its own species, man begins, using natural things and with the help of this nature, to create an infinity of species.

2. Application of graphene as electrical sensor (Field Effect Transistors):
Graphene is one of the most well studied nanomaterial in the field of nanotechnology [2]. Researchers find keen interest towards graphene and its derivatives and it has been studied for past 40 years [3-9]. Experimentally it has been found that a very large theoretical area of $2630 \mathrm{~m} 2 \mathrm{~g}-1$, the intrinsic mobility of $200000 \mathrm{~cm} 2 \mathrm{v}-1 \mathrm{~s}-1[10,11]$ and young modules value around $1.0 \mathrm{TPa}[12]$ has been shown by graphene. The thermal conductivity of graphene is around 5000 $\mathrm{Wm}-1 \mathrm{~K}-1[13,14]$ so both the electrical and thermal conductivity of graphene is high, makes the material appropriate for transparent conductive electrodes [15].

Graphene has been selected as one of the best material for the future generation electronic devices due to its 
unique 2D honeycomb lattice structure responsible for its zero gate semiconductor nature. The most important application of graphene is related to FET. A graphene based FET consists of one gate, a graphene channel connecting source and drain electrodes, and a dielectric barrier of $~ 300$ $\mathrm{nm} \mathrm{SiO}_{2}$ layer separating the gate from the channel and graphene as a dielectric layer in back gated graphene based FET [16]. Whereas, top gated graphene based MOSFETs have been produced with the help of exfoliated graphenes [17-20], chemical vapour deposition (CVD) based graphene grown on $\mathrm{Ni}$ and $\mathrm{Cu}$ substrates [21] and epitaxial graphene [22, 23] and the dielectric materials used for top gated grapehene based FET are $\mathrm{Al}_{2} \mathrm{O}_{3}, \mathrm{SiO}_{2}$ and $\mathrm{HfO}_{2}$ [24]. Back gated graphene FET devices have been investigated for years by several research groups [25-28]. A large scale transistor arrays with uniform electrical properties can be achieved by batch fabrication of graphene transistors with graphene grown on $\mathrm{Cu}$ films [29]. Top gated graphene based FET can be operated upto a frequency of $26 \mathrm{~Hz}$ [30]. The device mobility can be further changed by using an organic polymer buffer layer between graphene and conventional gate dielectrics. $100 \mathrm{~Hz}$ cut off frequency can be obtained by using an epitaxial graphene formed on a 2 inch $\mathrm{SiC}$ wafer [31]. De Heer group has found that mobility of graphene grown on the $\mathrm{Si}$ terminated face is less than that of carbon terminated face and this can help in gating [32]. The energy gap in graphene layer which is present just above the carbon buffer layer is $0.26 \mathrm{eV}[33,34]$, can be reduced with increasing thickness and becomes nearly zero when number of layers exceeds four [33]. The change in energy on going from single layer $(0.26$ $\mathrm{eV})$ to bilayer and trilayer $(0.14 \mathrm{eV})$ is simply attribute to the breaking of symmetry due interaction with the substrate explained with help of Angleresolved photoemission spectroscopy (ARPES) studies [35]. FET device made of pristine graphene shows low $\mathrm{I}_{\mathrm{on}} / \mathrm{I}_{\mathrm{off}}$ ratios due to the finite minimum conductance of graphene [25]. A bilayer insulating FET device can be made of an extra top gate along with a global back gate [36]. At a lower temperature this double gated graphene based FETs open a very small band gap of energy $10 \mathrm{meV}$ in the presence of a perpendicular electric field [37]. Whereas at room temperatures similar type of double gated graphene based FETs and infrared microspectroscopy can show a tunable band gap of energy $0.25 \mathrm{eV}$ in bilayer graphene based FETs [38]. A fabricated GNR FETs can be created by EBL patterning, coupled with oxygen plasma 
etching with widths between 10 and 100 $\mathrm{nm}$ and a band gap of $0.2 \mathrm{eV}$ has been observed by using a ribbon of width $15 \mathrm{~nm}$ [39]. Using the same device $I_{\text {on }} / I_{\text {offratio of }}$ $10^{4}$ is obtained [40]. Recently a chemical procedure has been followed to successful formation of GNR FETs with a ribbon width of $10 \mathrm{~nm}$ [41]. From the narrowest ribbon of width $2 \mathrm{~nm}$ the highest ratio of $\mathrm{I}_{\mathrm{on}} / \mathrm{I}_{\mathrm{off}} 10^{7}$ has been found [41]. Graphenebased FETs production can lead to development in research for the production of GNR FETs by 'unzipped' CNTs using plasma etching [42] or chemical oxidization [43].

A graphene $\mathrm{p}-\mathrm{n}$ junction has been produced in which the carrier type and the density of two adjacent regions decided by the electrostatic gating [44]. At the bipolar region a fractional conductance quantization has found experimentally [44] and the same can be explained theoretically [45]. $\mathrm{p}$ doped graphenes are in generally more common due to presence of adsorbate like water. For the preparation of $\mathrm{n}$ doped graphenes high power electrical annealing of GNRs [46] or high temperature annealing of graphene oxide [47] in ammonia $\left(\mathrm{NH}_{3}\right)$ is required. A p type GNR FET gives about $10^{5} \mathrm{I}_{\text {on }} / \mathrm{I}_{\text {off }}$ ratio after annealing [46]. Electrochemical doping can be achieved by adding an electrochemical top gate on the graphene FETand a doping level of up to $5 \times 10^{13}$ $\mathrm{cm}^{-2}$ has been found [48]. A controllable p-doping in graphene can be achieved by Surface modification of epitaxial graphene grown on $6 \mathrm{H}-\mathrm{SiC}(0001)$ with appropriate molecular acceptors [49].

\section{Table 1}

In the below table different types of substrates are analyzed to check their respective gate voltage and range of detection

\begin{tabular}{|l|l|l|l|l|}
\hline $\begin{array}{l}\text { Type of } \\
\text { substrate }\end{array}$ & $\begin{array}{l}\text { Detected } \\
\text { material }\end{array}$ & $\begin{array}{l}\text { Gate voltage } \\
(\mathbf{V})\end{array}$ & $\begin{array}{l}\text { Range of } \\
\text { detection }\end{array}$ & Reference \\
\hline $\mathrm{Cu}$ & $\mathrm{NH}_{3}, \mathrm{CO}_{2}$ & $>1$ & $\begin{array}{l}30 \mathrm{ppm}, 4000 \\
\mathrm{ppm}\end{array}$ & {$[50]$} \\
\hline $\begin{array}{l}\mathrm{N}-\mathrm{type} \mathrm{silicon} \\
\text { wafer }\end{array}$ & $\mathrm{NO}_{2}, \mathrm{NH}_{3}$ & $-5-5$ & $10 \mathrm{ppm}$ & {$[51]$} \\
\hline $\mathrm{Cu}$ & $\mathrm{NH}_{3}$ & 15 & $50 \mathrm{ppm}$ & {$[52]$} \\
\hline $\mathrm{SiO} / \mathrm{Si}$ & $\mathrm{H}_{2}$ & 0 & $1 \mathrm{ppm}$ & {$[53]$} \\
\hline $\mathrm{Glass}$ & $\mathrm{pH}$ & $-0.2-0.4$ & $3-10$ & {$[54]$} \\
\hline
\end{tabular}




\begin{tabular}{|c|c|c|c|c|}
\hline $\begin{array}{l}\mathrm{SiO}_{2} / \text { adhesive } \\
\text { tape }\end{array}$ & $\mathrm{K}^{+}$ & $-0.3-0.3$ & $10 \mathrm{nM}-1.0 \mathrm{mM}$ & [55] \\
\hline $\mathrm{SiO}_{2}$ & $\mathrm{Hg}^{2+}$ & $-20-20$ & $1 \mathrm{nM}$ & [57] \\
\hline PDMS & $\mathrm{Ca}^{2+}$ & $-0.6-0.6$ & $1 \mathrm{M}$ & [58] \\
\hline $\begin{array}{l}\text { Silicone } \\
\text { rubber/Glass }\end{array}$ & $\mathrm{Pb}^{2+}$ & $0-1$ & $0.02 \mathrm{~g} / \mathrm{L}$ & [59] \\
\hline
\end{tabular}

\section{Conclusion:}

Graphene has chosen as an efficient material for using it in various fields due to its unique structural features. The application of developing graphene as a sensor was a real challenge taken by the researchers and some of them were successful for the formation of totally graphene based sensors [60-62]. Although, this paper only covered few of the application of graphene but a broader way can be opened by using multilayeredgraphene except mono layer graphene sheet. Electrical sensors of graphene is quite popular due to its quantum capacitance effect that can control the capacitance with respect to the charge on the connection. Thus graphene based sensor can act as a wireless sensing system [63]. Graphene can act as a supercapacitor due to low band gap of valence and conduction band which makes it perfect for using it as electrochemical sensor [64-67]. From the above information it is very much clear that there are a lot of scopes of research on graphene and its oxide to make it better for making it more beneficial.

\section{References:}

[1] Martin, C.R. and Kohli, P., Nat. Rev. Drug Discov. 2, 29-37(2003)

[2] A. K. Geim , K. S. Novoselov , Nat. Mater. 6 ,183 (2007)

[3] H. P. Boehm, A. Clauss, G. O. Fischer, U. Hofmann, Z. Anorg. Allg. Chem. 316, 119 (1962)

[4] H. P. Boehm , R. Setton , E. Stumpp , Pure Appl. Chem., 66 , 1893 (1994) .

[5] H. P. Boehm , R. Setton , E. Stumpp , Carbon, 24 , 241 (1986) .

[6] J. Wintterlin , M. L. Bocquet, Surf. Sci. , 603, 1841 (2009) . 
[7] A. J. Vanbommel, J. E. Crombeen, A. Vantooren, Surf. Sci., 48,463 (1975) .

[8] X. K. Lu , M. F. Yu , H. Huang, R. S. Ruoff , Nanotechnology, 10, 269 (1999) .

[9] X. K. Lu , H. Huang, N. Nemchuk, R.

S. Ruoff , Appl. Phys. Lett., 75, 193 (1999)

[10] K. I. Bolotin , K. J. Sikes , Z. Jiang , M. Klima , G. Fudenberg, J. Hone, P. Kim , H. L. Stormer, Solid State Commun., 146 , 351 (2008) .

[11] S. V. Morozov, K. S. Novoselov, M. I. Katsnelson , F. Schedin , D. C. Elias , J. A. Jaszczak , A. K. Geim , Phys. Rev. Lett., 100, 016602 (2008)

[12] C. Lee , X. D. Wei , J. W. Kysar , J. Hone , Science, 321, 385 (2008)

[13]ShuaiWang,PriscillaKailianAng, Ziqian Wang, Ai Ling Lena Tang, John T.

L. Thong and Kian Ping Loh. Nano Lett. 10, 92-98 (2010).

[14] A. A. Balandin, S. Ghosh, W. Z. Bao , I. Calizo, D. Teweldebrhan, F. Miao, C. N. Lau , Nano Lett., 8 , 902 (2008)

[15] X. Li , Y. Zhu , W. Cai , M. Borysiak , B. Han, D. Chen, R. D. Piner, L. Colombo , R. S. Ruoff , Nano Lett., 9 , 4359 (2009)

[16] Virendra Singh, DaehaJoung, Lei Zhai, Soumen Das, Saiful I. Khondaker
,Sudipta Seal, Progress in Materials Science 56, 1178-1271(2011)

[17] Lemme MC,Echtermeyer TJ, Baus M, Kurz H, IEEE Electron DevLett; 28, 282 (2007)

[18] Liao L, Bai J, Qu Y, Lin Y-c, Li Y, Huang Y, Proceedings of the national academy of sciences,(2010)

[19] Lin Y-M, Jenkins KA, Valdes-Garcia A, Small JP, Farmer DB, Avouris P.,Nano Lett , 9, 422 (2008)

[20] Liao L, Bai J, Cheng R, Lin Y-C, Jiang S, Huang Y, Nano Lett, 10, 1917 (2010)

[21] Li X, Cai W, An J, Kim S, Nah J, Yang D,. Science, 324, 1312 (2009)

[22] Kedzierski J, Hsu P-L, Healey P, Wyatt PW, Keast CL, Sprinkle M, IEEE Trans Electron Dev, 55, 2078 (2008)

[23] Lin Y-M, Dimitrakopoulos C, Jenkins KA, Farmer DB, Chiu H-Y, Grill A, Science, 327, 662 (2010)

[24] Zhang Y, Tang T-T, Girit C, Hao Z, Martin MC, Zettl A, Nature, 459, 820 (2009)

[25] K. S. Novoselov, A. K. Geim , S. V. Morozov, D. Jiang, Y. Zhang, S. V. Dubonos , I. V. Grigorieva, A. A. Firsov, Science, 306, 666 (2004) 
[26] K. S. Novoselov, A. K. Geim , S. V. Morozov, D. Jiang, M. I. Katsnelson, I. V. Grigorieva, S. V. Dubonos, A. A. Firsov , Nature, 438 , 197 (2005)

[27] Y. B. Zhang , Y. W. Tan , H. L. Stormer , P. Kim , Nature, 438 ， 201 (2005)

[28] H. B. Heersche, P. Jarillo-Herrero, J. B. Oostinga , L. M. K. Vandersypen, A. F. Morpurgo , Nature, 446 , 56 (2007)

[29] M. P. Levendorf , C. S. Ruiz-Vargas , S. Garg , J. Park , Nano Lett. (2009)

[30] Y. M. Lin , K. A. Jenkins, A. ValdesGarcia , J. P. Small, D. B. Farmer , P. Avouris , Nano Lett, 9 , 422. (2009)

[31] Y.-M. Lin , C. Dimitrakopoulos , K. A. Jenkins, D. B. Farmer, H.Y.Chiu , A. Grill, P. Avouris , Science, 327 , 662 . (2009)

[32] De Heer WA, Berger C, Wu X, First PN, Conrad EH, Li X, Solid State Commun, 143, 92 (2007)

[33] Zhou SY, Gweon GH, Fedorov AV, First PN, deHeer WA, Lee DH, Nat Mater, 6, $770(2007)$

[34] Kim S, Ihm J, Choi HJ, Son Y-W, Phys Rev Lett, 100, 176802 (2008)

[35] S. Y. Zhou , G. H. Gweon , A. V. Fedorov, P. N. First, W. A. De Heer, D.
H. Lee, F. Guinea, A. H. C. Neto , A. Lanzara , Nat. Mater., 6, 770 (2007)

[36] J. B. Oostinga , H. B. Heersche, X. L. Liu , A. F. Morpurgo , L. M. K. Vandersypen, Nat. Mater, 7 , 151 (2008)

[37] Yanwu Zhu , ShanthiMurali , WeiweiCai, Xuesong Li , Ji Won Suk, Jeffrey R. Potts and Rodney S. Ruoff, Adv. Mater., 22, 3906-3924 (2010)

[38] Y. B. Zhang, T. T. Tang, C. Girit, Z. Hao , M. C. Martin , A. Zettl, M. F. Crommie, Y. R. Shen, F. Wang, Nature, $459,820(2009)$

[39] M. Y. Han , B. Ozyilmaz , Y. B. Zhang , P. Kim , Phys. Rev. Lett., 98 , 206805 (2007)

[40] Z. Chen, Y.-M. Lin, M. J. Rooks , P. Avouris , Phys. E, 40 , 228 (2007)

[41] X. L. Li , X. R. Wang, L. Zhang, S. W. Lee , H. J. Dai , Science, 319 , 1229 (2008)

[42] L. Y. Jiao , L. Zhang, X. R. Wang, G. Diankov, H. J. Dai , Nature ,458, 877 (2009)

[43] D. V. Kosynkin , A. L. Higginbotham , A. Sinitskii , J. R. Lomeda , A. Dimiev, B. K. Price , J. M. Tour , Nature, 458, 872 (2009) 
[44] J. R. Williams , L. DiCarlo , C. M. Marcus , Science, 317 , 638 (2007)

[45] D. A. Abanin , L. S. Levitov, Science , 317, $641(2007)$

[46] X. R. Wang, X. L. Li , L. Zhang, Y. Yoon, P. K. Weber, H. L. Wang, J. Guo, H. J. Dai , Science, 324 , 768 (2009)

[47] X. Li , H. Wang, J. T. Robinson, H. Sanchez, G. Diankov, H. Dai, J. Am. Chem. Soc. (2009)

[48] A. Das , S. Pisana , B. Chakraborty , S. Piscanec, S. K. Saha, U. V. Waghmare , K. S. Novoselov, H. R. Krishnamurthy, A. K. Geim , A. C. Ferrari , A. K. Sood, Nat. Nanotechnol., 3 , 210 (2008)

[49] W. Chen, S. Chen, D. C. Qi , X. Y. Gao , A. T. S. Wee , J. Am. Chem. Soc., 129, 10418 (2007)

[50] A. Inaba, G. Yoo, Y. Takei, K. Matsumoto, I. Shimoyama, IEEE 26th International Conference on, IEEE, pp. 969-972 (2013)

[51] C.-H. Kim, S.-W.Yoo, D.-W.Nam, S. Seo, J.-H. Lee, IEEE Electr. Device Lett., 33,1084-1086 (2012)

[52] M. Gautam, A.H. Jayatissa, ,J. Appl. Phys., 112, 064304 (2012)
[53] Z. Zhang, X. Zou, L. Xu, L. Liao, W. Liu, J. Ho, Nanoscale 7, 10078-10084 (2015)

[54] W. Fu, C. Nef, A. Tarasov, M. Wipf, R. Stoop, O. Knopfmacher, Nanoscale, 5 ,12104-12110 (2013)

[55] K. Maehashi, Y. Sofue, S. Okamoto, Y. Ohno, K. Inoue, K. Matsumoto, Sens. Actuators B, 187,45-49 (2013)

[56] T. Zhang, Z. Cheng, Y. Wang, Z. Li, C. Wang, Y. Li, Nano Lett., 10 ,47384741 (2010)

[57] H.G. Sudibya, Q. He, H. Zhang, P. Chen, ACS Nano, 5, 1990-1994 (2011)

[58] Y. Wen, F.Y. Li, X. Dong, J. Zhang, Q. Xiong, P. Chen, Adva. Healthcare Mater., 2,271-274 (2013)

[59] F. Schedin, A. K. Geim, S. V. Morozov , E. W. Hill , P. Blake, M. I. Katsnelson, K. S. Novoselov , Nat. Mater., $6,652(2007)$

[60] M.S. Mannoor, H. Tao, J.D. Clayton, A. Sengupta, D.L. Kaplan, R.R. Naik, Nat. Commun. 3,763 (2012)

[61] W. HyungáCheong, J. HyebáSong, J. JoonáKim, Wearable, Nanoscale 8,1059110597 (2016)

[62] S.J. Koester, Appl. Phys. Lett. 99, 163105 (2011) 
[63] D.A. Deen, E.J. Olson, M.A. Ebrish, S.J. Koester, IEEE Sens. J. 14,1459-1466 (2014)

[64] Z.S. Wu, Z. Liu, K. Parvez, X. Feng, K. Müllen, Adv. Mater. 27,3669-3675 (2015)

[65] D.A. Brownson, C.E. Banks, Chem. Commun. 48,1425-1427 (2012)

[66] E.O. Polat, C. Kocabas, Nano Lett. 13,5851-5857 (2013)

[67] J.J. Yoo, K. Balakrishnan, J. Huang, V. Meunier, B.G. Sumpter, A. Srivastava, Nano Lett. 11,1423-1427 (2011) 